\title{
Neuroglobin Provides a Convenient Scaffold to Investigate the Triplet-State Properties of Porphyrins by Time-Resolved EPR Spectroscopy and Magnetophotoselection
}

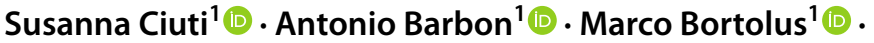 \\ Alessandro Agostini ${ }^{1,3}$ (ㄷ) Elisabetta Bergantino ${ }^{2}$ (ㄷ) Caterina Martin ${ }^{2}$. \\ Marilena Di Valentin ${ }^{1,4}$ (i) $\cdot$ Donatella Carbonera ${ }^{1,4}(\mathbb{C}$
}

Received: 15 June 2021 / Revised: 18 August 2021 / Accepted: 19 August 2021 /

Published online: 4 September 2021

(c) The Author(s) 2021

\begin{abstract}
The photo-excited triplet state of Zn-protoporphyrin IX located in the heme pocket of human neuroglobin has been investigated by time-resolved EPR coupled to magnetophotoselection. The triplet state in the protein matrix has been compared with the model complex in organic glass, considering both non-coordinating and coordinating solvent mixtures. The protein matrix plays an important role in stabilizing the coordination of the embedded chromophore, resulting in a more homogeneous environment relative to that of the chromophore in a glassy solvent, even in the presence of an axial nitrogenous ligand like pyridine. The EPR spectral parameters point out a slow Jahn-Teller interconversion between slightly different triplet states, both in organic solvent and in the protein matrix. The EPR-magnetophotoselection allows us to propose a reinterpretation of the assignment of the $\mathrm{Q}$ bands in the electronic absorption spectrum.
\end{abstract}

Marilena Di Valentin

marilena.divalentin@unipd.it

$\bowtie$ Donatella Carbonera

donatella.carbonera@unipd.it

1 Department of Chemical Sciences, University of Padova, via Marzolo 1, 35131 Padua, Italy

2 Department of Biology, University of Padova, via Bassi 58/b, 35131 Padua, Italy

3 Biology Centre, Institute of Plant Molecular Biology, Czech Academy of Sciences, Branišovská 31, 37005 Ceske Budejovice, Czech Republic

4 Interdepartmental Centre Giorgio Levi Cases for Energy Economics and Technology, University of Padova, via Marzolo 9, 35131 Padua, Italy 


\section{Introduction}

Porphyrins are dubbed the "pigments of life" in virtue of their ubiquitous presence in all three domains of life, playing fundamental roles in catalysis, regulation, transport, respiration, and photosynthesis [1]. Because of the properties of their excited states, porphyrins have been considered for many applications, such as artificial photosynthesis and organic photovoltaics [2]. The structure of these molecules is characterized by a tetrapyrrolic macrocycle, and subtle molecular modifications (pyrrole reduction, metalation and other derivatization) tune electronic and functional properties of porphyrins [3].

The photo-excited triplet states of this class of molecules are of special interest, due to their capability to photosensitize singlet oxygen with high quantum yields, leading to an extensive use as photosensitizers for photodynamic therapy [4-6]. Despite this applicative interest, an in-depth characterization of the triplet state is still lacking due to the complex chemistry and photophysics of porphyrins. The investigation in vitro is hindered by their tendency to form aggregates, being tetrapyrroles characterized by strong $\pi-\pi$ interactions [7]. Moreover, metalloporphyrins show a marked tendency to self-chelate the central metal ion to coordinating side groups [8]. Due to these properties, oligomerization is likely to take place and has been observed also in the naturally occurring chlorosomes [9] and hemozoin [10]. In addition, metalloporphyrins may coordinate solvent molecules to the central metal in a five/six coordination mode, depending on the metal and on the solvent, thus increasing the number of different species present in solution [11, 12]. The analysis of the optical and magnetic spectra of porphyrins is often complicated by the abovementioned effects. On the other hand, a full characterization of the isolated monomer properties is required also as firm starting input for the quantitative analysis of singlet and/or triplet states in multimeric systems where excitonic states, which are important for the functions played, are often formed [13-17].

To investigate tetrapyrroles in monomeric and chemically well-defined forms, a convenient approach is to insert them in tetrapyrrole-binding proteins $[18,19]$, such as those belonging to the globin class. These all-alpha proteins are watersoluble and bind a single porphyrin in a conserved pocket (Fig. 1B), providing a defined molecular surrounding to the chromophores and preventing their aggregation. Human neuroglobin have been recently proposed as a convenient platform for spectroscopic investigations, in virtue of its high-resolution crystallographic structure [20], monomeric assembly, and feasibility of porphyrin substitution [21]: the porphyrin bound by the protein has been used also as internal probe for distance measurements by means of double electron-electron resonance [22-24].

The triplet state of Zn-Protoporphyrin IX (ZnPP, Fig. 1A), inserted in the heme pocket of neuroglobin (Fig. 1B), has been investigated by Time-Resolved EPR (TREPR) spectroscopy coupled to magnetophotoselection experiments, as the first step in the selection of a suitable protein scaffold to be further used for triplet-state characterization of other monomeric porphyrins, such as Mg-Protoporphyrin IX and chlorophyllide, in the perspective of having a reliable model system for the interpretation of triplet states of complex multimeric porphyrin assemblies [14, 25, 26]. 

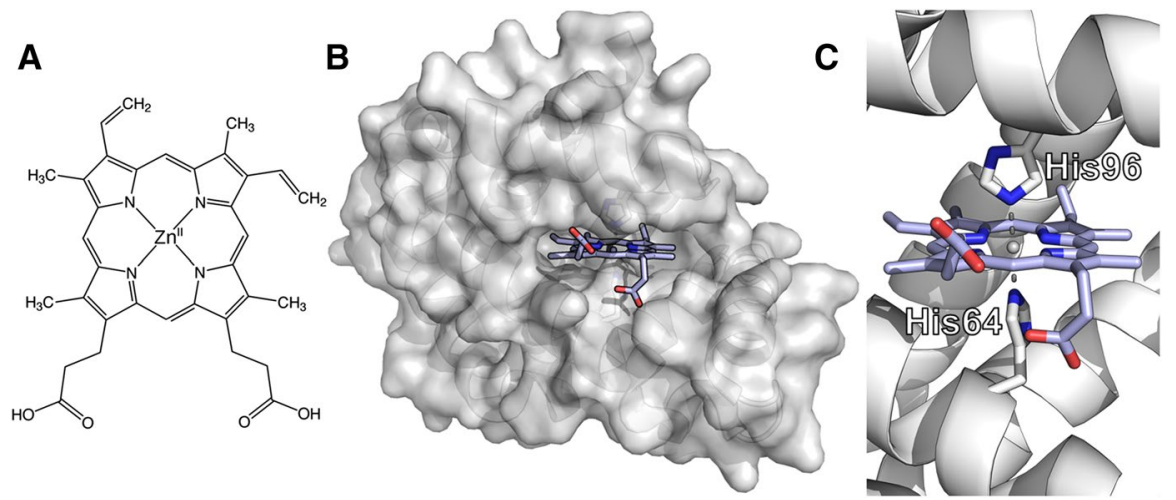

Fig. 1 A Structure of Zn-Protoporphyrin IX $(Z n P P)$. B Crystallographic structure of human neuroglobin (PDB: 4MPM [20]). In this work, the naturally occurring Fe(II)-Protoporphyrin IX cofactor has been substituted by $Z n P P$. C Heme binding pocket

In the study of the photo-excited triplet states, magnetophotoselection experiments provide unique information, allowing determination of the orientation of the zero field splitting (ZFS) tensor, which describes the interaction of the two unpaired electron spins of the triplet state, relative to frame of the optical transition dipole moment (TDM) of the molecule [27-29]. Linearly polarized excitation light (parallel/perpendicular to the external magnetic field) is used to produce an orientation selection effect, which is reflected by a specific change in the intensities of the EPR transitions. The TDM corresponding to the Soret and Q bands, which can be degenerate when the molecular symmetry is $\mathrm{D}_{4 \mathrm{~h}}$, are located in the tetrapyrrole molecular plane $[3,30]$.

Metalloporphyrins possess a $\mathrm{D}_{4 \mathrm{~h}}$ symmetry in the four-orbital approximation [30, 31], which should lead to the lowest excited triplet state being doubly degenerate. Moreover, under $\mathrm{D}_{4 \mathrm{~h}}$ symmetry, the ZFS tensor is characterized by axial symmetry. The degeneracy of the triplet state may be lifted by Jahn-Teller distortion of the plane of the molecule and/or asymmetry of the environment [32]. Such an effect also removes the axial symmetry of the ZFS tensor and leads to opposite values of the ZFS parameter E in the two states. Depending on the temperature and on the energy difference between these two states, back and forth transitions between them may occur $[33,34]$.

In this work, we show that EPR-magnetophotoselection can help in recognizing the presence of a complex behavior in the triplet state of metalloporphyrins. We analyze the results obtained in the study of $Z n P P$ in recombinant human neuroglobin, we compare them with those obtained in non-coordinated and coordinated solvent and discuss the triplet-state properties in terms of the Jahn-Teller effect while proposing a reinterpretation of the Q-band region of the electronic absorption spectrum. 


\section{Experimental}

\subsection{Sample Preparation}

ZnPP and all solvents (2-Methyltetrahydrofuran, MeTHF; dichloromethane, DCM; pyridine) were purchased from Sigma Aldrich and used as received. ZnPP was dissolved in a MeTHF:DCM 3:1 solvent mixture preparing a $1 \mathrm{mM}$ stock solution and then diluting it down to $100 \mu \mathrm{M}$ in the same solvent mixture while adding the desired vol\% of pyridine.

The human neuroglobin DNA coding sequence, cloned in the vector pET3a, was kindly donated by Professor T. Burmester (Department of Biology, University of Hamburg). The expression and purification of human neuroglobin have been performed as previously reported in great detail [24]. The ZnPP-substitution was performed following the procedure described by Scholler et al. [21], performing all manipulations in the dark because of the light sensitivity of ZnPP. In brief, ZnPP was solubilized in $0.1 \mathrm{M} \mathrm{NaOH}$ and dissolved in buffer $5 \mathrm{mM}$ Tris- $\mathrm{HCl} \mathrm{pH}$ 8.5. This was added to the apo-neuroglobin solution, which was then incubated for $4 \mathrm{~h}$, dialyzed overnight against $5 \mathrm{mM}$ Tris- $\mathrm{HCl}$ buffer at $\mathrm{pH} 8.5$ and concentrated by Amicon filtration (PM10). The yield of $Z n P P$-substituted neuroglobin from apo-protein resulted to be $50 \%$ (measured by $Z n P P$ absorbance using $\varepsilon_{424 \mathrm{~nm}}=122 \mathrm{mM}^{-1} \mathrm{~cm}^{-1}$ ). The residual apo-protein, remaining denaturated or mis-folded, was easily eliminated by centrifugation. Glycerol, previously degassed by several cycles of freezing and pumping, was added (60\% v/v) just before freezing to obtain a transparent matrix. The final sample concentration was $250 \mu \mathrm{M}$.

\subsection{UV-Vis Spectroscopy}

Absorption spectra were acquired using two matched quartz cuvettes with a $1 \mathrm{~mm}$ optical path on a Lambda 35 spectrometer by PerkinElmer. The absence of ZnPP aggregation was evaluated by inspecting the absorption spectrum at progressive dilutions from 100 down to $10 \mu \mathrm{M}$ : no shifts in the absorption bands nor in their relative intensities were present.

\subsection{TR-EPR Experiments}

TR-EPR experiments were performed on a Bruker ELEXSYS E580 spectrometer, equipped with a dielectric resonator (Bruker ER 4117-DI5, TE ${ }_{011}$ mode), an Oxford CF935 flow cryostat, and an Oxford ITC4 temperature controller. The microwave frequency was measured by a frequency counter, (HP5342A). The temperature in the cryostat was controlled by a thermostated nitrogen flow and all experiments were conducted at $80 \mathrm{~K}$, disabling magnetic field modulation. Photo-excitation was conducted with a Nd:YAG pulsed laser (Quantel Brilliant) equipped with both second and third harmonic modules and an optical parametric oscillator $(\mathrm{OPOTECH})$ (pulse length $=5 \mathrm{~ns}, \mathrm{E} / \mathrm{pulse} \cong 2 \mathrm{~mJ}, 10 \mathrm{~Hz}$ repetition 
time). The EPR direct-detected signal, after preamplification with a $6 \mathrm{MHz}$ amplifier, was recorded with a LeCroy 9300 digital oscilloscope, triggered by the laser pulse. For every field position, 100-200 transient signals were averaged. Two different polarizations of the light were employed for the magnetophotoselection experiments: one with optical polarization perpendicular and the other parallel to the static magnetic field of the spectrometer. The rotation of the polarization plane of the light was obtained using a broadband half waveplate; a linear polarizer was added near the optical window of the resonator for a better control of the polarization.

\subsection{Spectral Analysis}

TR-EPR data were processed by subtracting: (i) the average baseline before the trigger event and (ii) a time profile taken at an off-resonance position to eliminate the laser background signal. Spectra were extracted as averaging $100 \mathrm{~ns}$ of the transient signal at about 1.2-1.4 $\mu$ s after pulsed laser excitation. Simulations of triplet spectra have been performed using a home-written MATLAB program which includes fulldiagonalization of the spin Hamiltonian [27, 35]. For the isotropically excited TREPR spectrum, the parameters of the simulations include the $g$ and ZFS tensors and the relative triplet sublevel populations $\left(p_{\mathrm{x}}, p_{\mathrm{y}}\right.$, and $\left.p_{\mathrm{z}}\right)$.

\section{Results and Discussion}

We initially performed TR-EPR on the ZnPP in organic glass to gain information on the electronic structure of the excited triplet state and compare the results obtained for non-coordinating and coordinating solvents. Figure 2A displays the electronic absorption spectrum in the MeTHF:DCM solvent mixture with increasing amounts of pyridine and Fig. 2B the corresponding X-band TR-EPR spectrum of the triplet state of $\mathrm{ZnPP}$ at $80 \mathrm{~K}$ with isotropic excitation at $580 \mathrm{~nm}$, on the maximum of one of the two $\mathrm{Q}$ bands.

To ensure isotropic excitation conditions, the TR-EPR spectrum is calculated as the sum of the experimental spectrum recorded after excitation with polarization of the light parallel to the external magnetic field and twice the spectrum obtained with perpendicular polarization, in analogy to optical polarization measurements. The EPR spectrum shows a polarization pattern of absorptive and emissive lines (aaaeee), which is typical for $\mathrm{Zn}(\mathrm{II})$-porphyrin systems [36]. The turning points of the EPR spectrum yield the ZFS parameters D and E that describe the dipolar interaction of the two unpaired electron spins of the triplet state. The ZFS parameter D, which is positive for most porphyrin macrocycles [37] in absence of strong distortions [38], provides an estimate of the radial extension of the triplet-state wave function, while the parameter $\mathrm{E}$ is sensitive towards rhombic distortions of the porphyrin moiety. The corresponding values in the pyridine solvent are reported in Table 1 and match with those previously reported for $Z n P P$ in organic glass by continuous-wave EPR [39]. It must be noted that a simulation of the spectra with a single, defined 


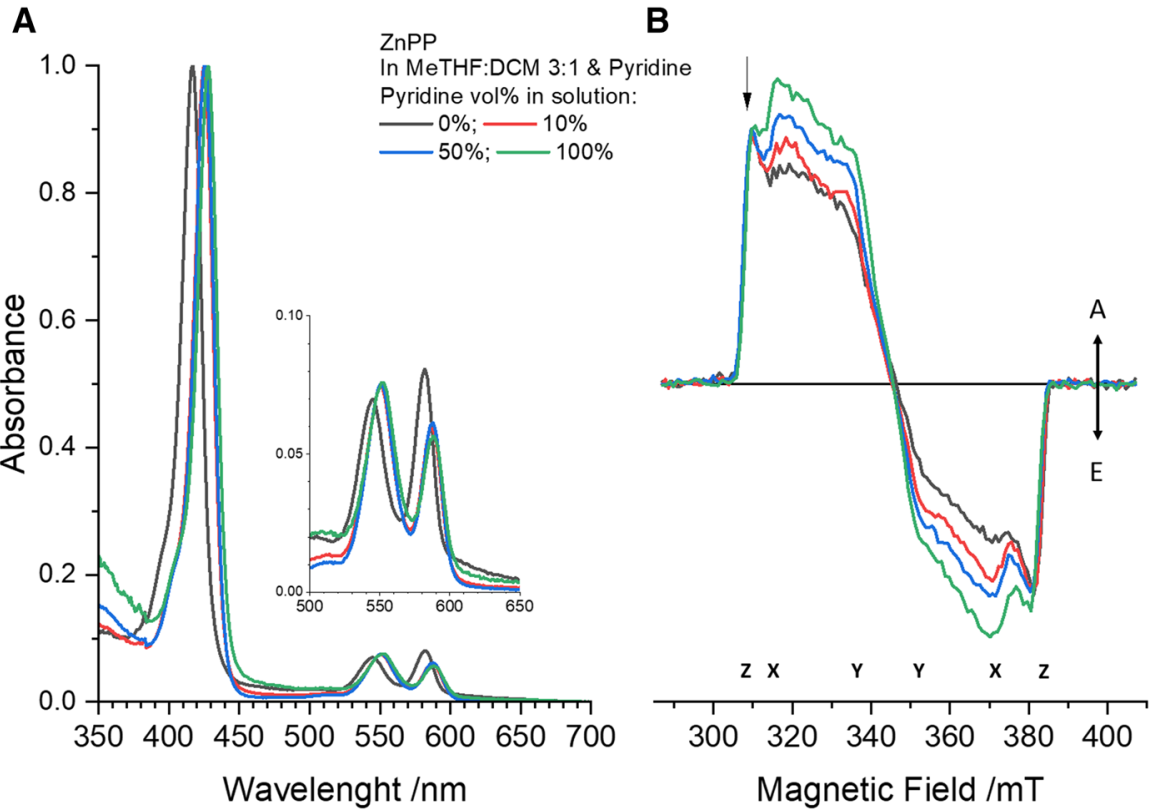

Fig. 2 A Normalized UV-Vis absorption spectra of $Z n P P$ (concentration $100 \mu \mathrm{M}$ ) in MeTHF:DCM 3:1 solvent mixture at increasing vol\% of pyridine: $0 \%$ (black); $10 \%$ (red); $50 \%$ (blue); $100 \%$ (pure pyridine-green). $\mathrm{T}=298 \mathrm{~K}$; B X-band TR-EPR spectra with isotropic excitation in the same solutions as $\mathbf{A}$. The arrows denote absorption (A) and enhanced emission (E); the positions of the principal ZFS components are indicated below the spectra. The TR-EPR spectra are normalized on the feature indicated by the arrow corresponding to the $\mathrm{Z}$ component of the ZFS tensor. $\lambda_{\text {exc }}=580 \mathrm{~nm} ; \mathrm{T}=80 \mathrm{~K}$

Table 1 Triplet-state parameters for ZnPP in neuroglobin and in MeTHF:DCM 3:1 with 50 vol\% pyridine solvent mixture derived from TR-EPR-magnetophotoselection at different excitation wavelengths

\begin{tabular}{|c|c|c|c|c|c|c|c|c|c|}
\hline & $\lambda(\mathrm{nm})$ & $D^{\mathrm{I}}\left(10^{-3} \mathrm{~cm}^{-1}\right)$ & $\mathrm{E}^{\mathrm{I}}\left(10^{-3} \mathrm{~cm}^{-1}\right)$ & $p_{\mathrm{x}}{ }^{\mathrm{I}}$ & $p_{\mathrm{y}}{ }^{\mathrm{I}}$ & $p_{\mathrm{z}}{ }^{\mathrm{I}}$ & $\mathrm{PR}_{\mathrm{x}}^{\mathrm{II}}$ & $\mathrm{PR}_{\mathrm{y}}^{\mathrm{II}}$ & $\mathrm{PR}_{\mathrm{z}}^{\mathrm{II}}$ \\
\hline \multirow[t]{2}{*}{ Protein } & 544 & +34.9 & -6.0 & & 0 & 0.98 & -0.17 & 0.13 & -0.66 \\
\hline & 580 & & & & & & -0.21 & 0.09 & -0.62 \\
\hline \multirow[t]{2}{*}{ Solvent } & 544 & +35.4 & -6.7 & & - & - & -0.03 & 0.15 & -0.18 \\
\hline & 580 & & & & & & -0.05 & 0.13 & -0.18 \\
\hline
\end{tabular}

IZFS parameters $\mathrm{D}$ and $\mathrm{E}\left( \pm 1 \times 10^{-4} \mathrm{~cm}^{-1}\right)$ and relative population rates $p_{\mathrm{x}}, p_{\mathrm{y}}$ and $p_{\mathrm{z}}( \pm 0.02)$ from simulation of the isotropically excited TR-EPR spectrum for ZnPP in neuroglobin. D and E parameters are derived directly from the turning points of the spectrum in the glass solvent (MeTHF:DCM 3:1 with $50 \mathrm{vol} \%$ pyridine). The signs of the ZFS parameters are discussed in the text

IIPolarization Ratio $\mathrm{PR}_{i}( \pm 0.01)$ calculated at the canonical transitions $i=x, y, z$, considering the absolute values of the intensities at the high-field turning points of the TR-EPR spectra in the magnetophotoselection experiment, according to Eq. 1

value of $\mathrm{E}$ is not feasible, even introducing $\mathrm{E}$ strains; for this reason, the values reported in Table 1 are not the output of the simulation but are taken directly at the turning points of the spectra. 
We observe an influence of the axial ligand on the absorption spectrum in terms of spectral shifts, in both the Soret and Q-band regions, as a function of the increased content of pyridine in the solvent mixture, in agreement with past experiments performed on $Z n P P$ [40]. According to the literature, the $\mathrm{Q}$ transitions have been tentatively assigned to either the two distinct $Q_{\mathrm{x}}$ and $Q_{\mathrm{y}}$ transitions or two vibronic transitions $Q(0-1)$ and $Q(0-0)$ for $\mathrm{Zn}(\mathrm{II})$ porphyrin systems [41-44]. From the point of view of the triplet-state spectrum, the spin-polarization pattern remains unaffected by increasing the amount of coordinating pyridine solvent, while the turning points corresponding to the $\mathrm{X}$ and $\mathrm{Y}$ transitions become more defined. The EPR spectrum is more sensitive than the electronic absorption spectrum to the increasing content of the axial ligand and it is continuously changing with the pyridine content. This behavior may be indicative of strain effects on the $E$ parameter due to an inhomogeneous coordination environment, which affects only the $X$ and $Y$ transitions since the $Z$ transitions are separated by 2D. Alternatively, the spectral broadening could be ascribed to a slow Jahn-Teller interconversion rate between slightly different triplet states. Similar behavior was reported in triplet spectra recorded by continuous-wave EPR [39].

Figure $3 \mathrm{~A}$ shows the $\mathrm{X}$-band TR-EPR spectrum of the triplet state of $\mathrm{ZnPP}$ in neuroglobin at $80 \mathrm{~K}$ with isotropic excitation at $544 \mathrm{~nm}$, on the maximum of one of the $\mathrm{Q}$ bands, and the corresponding simulation. The electronic absorption spectrum, previously reported in [24], is characterized by the presence of two intense Q bands, as for the organic solvent.

Unlike the above-mentioned spectra in organic glass, a satisfactory simulation of the TR-EPR lineshape could be obtained; a better matching was reached by taking into account a small net spin-polarization contribution that was previously discussed in $[33,34]$. The simulation parameters are reported in Table 1 , the relative population rates and the rhombic ZFS tensor correspond to those previously derived for $Z n P P$ in neuroglobin [33] and in other protein scaffolds [18, 19]. The EPR linewidth is narrow compared with the glass solvent. We note that incorporation of ZnPP into apo-neuroglobin produces pronounced effects on the triplet-state properties as it exhibits an extremely well-resolved triplet-state spectrum. This indicates a specific binding of the cofactor in the protein environment. The ZFS tensor is rhombic and the value derived for the E parameter points out that the dynamical average due to the Jahn-Teller effect, if there, is not complete at the temperature of the experiments, since a lower E value would be expected in that case.

In the magnetophotoselection experiments, the sample is excited with linearly polarized light, either parallel or perpendicular to the magnetic field and the relative intensities of the EPR lines depend on the light polarization [27]. It is a consequence of the fact that the EPR intensities depend not only on the population difference between the triplet sublevels, but also on the non-uniform spatial distribution of the molecules due to selective photoexcitation. The TR-EPR spectra for the triplet state of $Z n P P$ with light polarized parallel and perpendicular to the magnetic field are compared both in Fig. 3B and C. Excitation wavelengths are $580 \mathrm{~nm}$ and at $544 \mathrm{~nm}$, respectively, on each of the two Q bands. Since the experimental setup assured the same excitation conditions, the spectra are displayed without renormalization and show an evident magnetophotoselection effect, with enhanced $X$ and $Y$ 
A

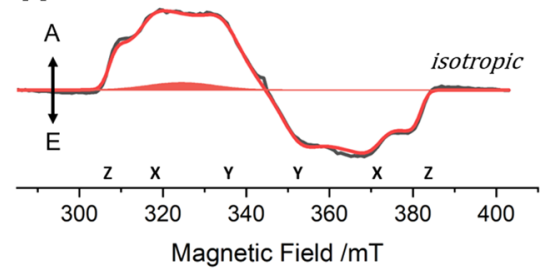

B
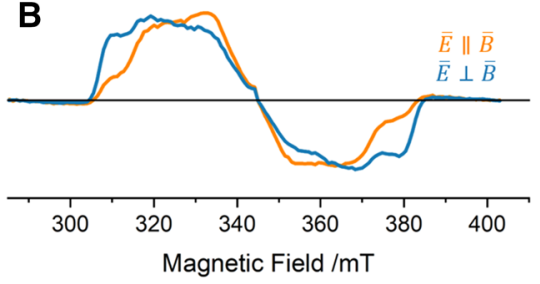

C

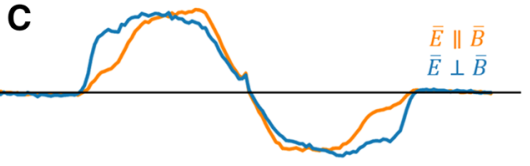

300
$320 \quad 340 \quad 360 \quad 380 \quad 400$
Magnetic Field /mT
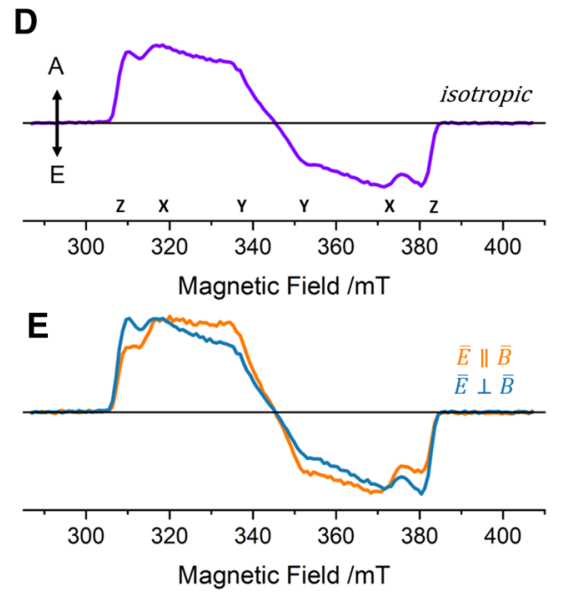

$\mathbf{F}$

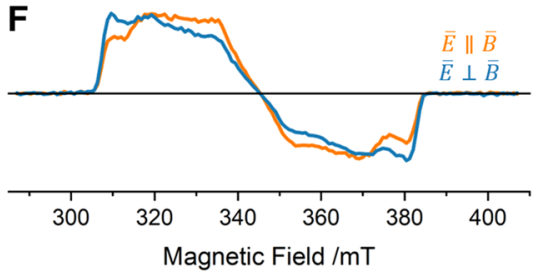

Fig. 3 X-band TR-EPR spectra of ZnPP reconstituted in neuroglobin (left panels) and in MeTHF:DCM 3:1 with 50vol\% pyridine (right panels) at $80 \mathrm{~K}$. A and D Spectrum with isotropic excitation, $\lambda_{\mathrm{exc}}=544 \mathrm{~nm}$ and simulation when present (red). The Gaussian net polarization contribution added to the simulation is represented in light red. B and $\mathbf{E}$ Magnetophotoselection spectra, $\lambda_{\text {exc }}=544 \mathrm{~nm}$. Laserpolarized parallel to the magnetic field (orange- $-\bar{E}|| \vec{B})$ ); laser-polarized perpendicular to the magnetic field (azure $-\bar{E} \perp \bar{B}$ ). $\mathbf{C}$ and $\mathbf{F}$ Magnetophotoselection, $\lambda_{\mathrm{exc}}=580 \mathrm{~nm}$. Laser-polarized parallel to the magnetic field (orange- $-\bar{E}|| \bar{B}$ ); laser-polarized perpendicular to the magnetic field (azure $-\bar{E} \perp \bar{B}$ ). The arrows denote absorption $(\mathbf{A})$ and enhanced emission $(\mathbf{E})$; the positions of the principal ZFS components are indicated below the isotropic spectrum

triplet transitions in the parallel spectrum and enhanced $Z$ transitions in the perpendicular spectrum. The magnetophotoselection effect does not show any significant wavelength dependence. This is an important experimental evidence that must be considered when discussing the potential presence of Jahn-Teller dynamic effects.

The spectral analysis allows to define the relative orientation between the optical TDM and the ZFS principal axes [27]. Magnetophotoselection, applied to porphyrin systems, has been used in a limited number of cases to attain quantitative information on the relative orientation of the ZFS tensor axes with respect to the TDM, if one of the two is known $[35,45]$. Spectral simulations require the correct formalism to take into account potential dynamic Jahn-Teller effects in the specific case of $Z n P P$. For this reason, work in this direction is currently underway. Alternatively, qualitative considerations can be discussed, focusing the attention on the observed absence of wavelength effects on the magnetophotoselection behavior.

It is possible to calculate from the TR-EPR spectra the polarization ratio (PR) defined as $[46,47]$ 


$$
\mathrm{PR}=\frac{\left(I_{\mathrm{par}}-I_{\text {perp }}\right)}{\left(I_{\mathrm{par}}+I_{\text {perp }}\right)},
$$

where $I_{\text {par }} / I_{\text {perp }}$ are the intensities of the EPR signal for excitation with light polarized parallel or perpendicular to the magnetic field at the field positions corresponding to the $X, Y$, or $Z$ orientation of the ZFS tensor.

If the optical TDM is approximately collinear with one of the ZFS tensor axes, the corresponding polarization ratio is expected to be positive, while the polarization ratios for the other two principal orientations are negative. It is found that for other $\mathrm{Zn}$ (II)porphyrin systems this condition is satisfied, and, varying the excitation on the two Q bands, the polarization ratio is found to be positive alternatively for the $X$ and $Y$ transitions, demonstrating that the in-plane ZFS components are parallel to the TDM of the $Q_{\mathrm{x}}$ and $Q_{\mathrm{y}}$ transitions [46]. For $Z n P P$ in neuroglobin, the polarization ratio is positive at both wavelengths in correspondence to the $Y$ axes (see Table 1). At the same time, the small dependence of the magnetophotoselection on the excitation wavelength does not allow a simple interpretation of the results. Even a rotation of the ZFS frame with respect to the optical axes, as reported for chlorophyll derivatives $[48,49]$, would not account for the magnetophotoselection results. An intermediate angle between the $X$ and $Y$ direction would explain the absence of wavelength dependence, but would not account for the polarization ratios that, although qualitative, point towards the collinearity between the TDM and $Y$ ZFS axis.

Interpretation of the magnetophotoselection experiments likely requires revisiting the assignment of the electronic absorption spectra in the $Q$ region where the two bands are not due to distinct $Q_{\mathrm{x}}$ and $Q_{\mathrm{y}}$ contributions, as expected for a porphyrin macrocycle with a reduced symmetry, but should be assigned to a degenerate $Q$-band, showing the $Q(0-0)$ and the $Q(0-1)$ vibronic progression. This question is indeed still under debate and clear experimental evidence for a correct assignment is still lacking [41-44]. Alternatively, a different interpretation can be proposed in the frame of the dynamic Jahn-Teller effect, which has been invoked to account for the net polarization effect in the TR-EPR spectrum of ZnPP in neuroglobin [33]. A complete dynamic averaging of the ZFS parameter E would correspond to a fast pseudo-rotation in the porphyrin plane and the magnetophotoselection should be indistinguishable while exciting on each of the two $Q$ bands. However, this does not apply to $Z n P P$ since some rhombicity is still present. Thus, the Jahn-Teller distortions alone cannot explain the experimental results. This conclusion is also suggested when comparing the results obtained in the organic solvent to those corresponding to the protein matrix.

A clear broadening is visible in the $X-Y$ region for the isotropic TR-EPR spectrum in solvent matrix (see Fig. 3D), as already pointed out in the previous sections, which might be due to a distribution of distorted configurations in the organic solvent. It is also likely that different vibronic triplet states, separated by energy gaps close to $\mathrm{K}_{\mathrm{B}} \mathrm{T}$, contribute to the spectra increasing the broadening because of the slow dynamic regime. The magnetophotoselection experiments in organic glass (see Fig. 3E and F), where fast dynamic averaging can be excluded, give similar results to those obtained in protein. The polarization ratio is positive again for the $Y$ transition, 
and no wavelength dependence is present (see Table 1). We can, therefore, be confident that magnetophotoselection experiments provide unambiguous evidence that the electronic spectrum in the $Q$-band region is due to a degenerate electronic transition showing the vibronic progression and the $Q_{\mathrm{x}}$ and $Q_{\mathrm{y}}$ components, being almost degenerate, are buried in the inhomogeneously broaden spectrum.

\section{Conclusions}

We have demonstrated that magnetophotoselection is a key spectroscopic tool in providing valuable information on the electronic structure of the porphyrin molecule in the excited states and assessing the corresponding symmetry. Combining optical and magnetic selection, the information is not confined to the paramagnetic triplet state, but it is extended to the corresponding excited singlet state connected by the intersystem crossing mechanism, as already highlighted in previous work in the case of free-base porphyrins, where a phototautomerization process is occurring [35]. The results provide motivation for further investigation to characterize the complex excited states behavior of metalloporphyrins.

The protein matrix plays an important role in stabilizing the coordination of the imbedded chromophore, resulting in a more homogeneous environment than in the case of a chromophore in a glassy solvent, even in the presence of an axial nitrogenous ligand like pyridine. In protein, Jahn-Teller distortions may still be present, but the number of states involved would be strongly reduced. When investigating the electronic properties of the porphyrin molecule, it is of fundamental importance to control the local structure surrounding the tetrapyrrole macrocycle to take into account specific interactions, which can be nowadays rigorously modeled by advanced computational methodologies [50, 51].

Funding Open access funding provided by Università degli Studi di Padova within the CRUI-CARE Agreement. Financial supports to D.C. (P-DiSC-2019), to M.D.V. (Biomolecular DSSCs Project from Interdepartmental Center Giorgio Levi Cases for Energy Economics and Technology), to M.B. (NExuS Project from Dipartimento d'Eccellenza) and to A.A. (institutional support RVO:60077344 and MEMOVA project, Eu Operational Programme Research, Development and Education no. CZ.02.2.69/0. 0/0.0/18_053/0016982) are gratefully acknowledged.

Data Availability All data needed to evaluate the conclusions in the paper are present in the paper.

\section{Declarations}

Conflict of Interest The authors declare no competing interests.

Open Access This article is licensed under a Creative Commons Attribution 4.0 International License, which permits use, sharing, adaptation, distribution and reproduction in any medium or format, as long as you give appropriate credit to the original author(s) and the source, provide a link to the Creative Commons licence, and indicate if changes were made. The images or other third party material in this article are included in the article's Creative Commons licence, unless indicated otherwise in a credit line to the material. If material is not included in the article's Creative Commons licence and your intended use is not permitted by statutory regulation or exceeds the permitted use, you will need to obtain permission 
directly from the copyright holder. To view a copy of this licence, visit http://creativecommons.org/licen ses/by/4.0/.

\section{References}

1. D. Dolphin, The Porphyrins, Part B: Biochemistry, vol. VII (Academic Press, New York, San Francisco, London, 1979), pp.1-529

2. J. Min Park, J.H. Lee, W.-D. Jang, Coord Chem. Rev. 407, 213157 (2020)

3. M. Gouterman, J. Mol. Spectrosc. 6, 138 (1961)

4. R.R. Allison, C.H. Sibata, Photodiagnosis Photodyn. Ther. 7, 61 (2010)

5. M. Ethirajan, Y. Chen, P. Joshi, R.K. Pandey, Chem. Soc. Rev. 40, 340 (2011)

6. A. Agostini, M.G. Dal Farra, H. Paulsen, A. Polimeno, L. Orian, M. Di Valentin, D. Carbonera, J. Phys. Chem. B 123, 8232 (2019)

7. C.A. Hunter, J.K.M. Sanders, J. Am. Chem. Soc. 112, 5525 (1990)

8. L. Fiedor, A. Kania, B. Myśliwa-Kurdziel, Ł Orzeł, G. Stochel, Biochim. Biophys. Acta Bioenerg. 1777, 1491 (2008)

9. J.M. Linnanto, J.E.I. Korppi-Tommola, Photosynth. Res. 96, 227 (2008)

10. A.F. Slater, W.J. Swiggard, B.R. Orton, W.D. Flitter, D.E. Goldberg, A. Cerami, G.B. Henderson, Proc. Natl. Acad. Sci. 88, 325 (1991)

11. J. W. Buchler, in The Porphyrins, vol. 1, ed. by D. Dolphin (Academic Press, New York, San Francisco, London, 1978), pp. 389-483

12. M. Gouterman, in The Porphyrins, vol. 3, ed. by D. Dolphin (Academic Press, New York, San Francisco, London,1978), pp. 1-165

13. H. van Amerongen, R. van Grondelle, L. Valkunas, Photosynthetic Excitons (World Scientific, Singapore, 2000)

14. A. Agostini, D.M. Palm, H. Paulsen, D. Carbonera, J. Phys. Chem. B 122, 6156 (2018)

15. C.E. Tait, P. Neuhaus, M.D. Peeks, H.L. Anderson, C.R. Timmel, J. Am. Chem. Soc. 137, 8284 (2015)

16. T. Renger, M.E.-A. Madjet, M. Schmidt am Busch, J. Adolphs, F. Müh, Photosynth. Res. 116, 367 (2013)

17. S. Kundu, A. Patra, Chem. Rev. 117, 712 (2017)

18. P.J. Angiolillo, J.M. Vanderkooi, Biophys. J. 68, 2505 (1995)

19. M. Fahnenschmidt, R. Bittl, E. Schlodder, W. Haehnel, W. Lubitz, Phys. Chem. Chem. Phys. 3, 4082 (2001)

20. B.G. Guimarães, D. Hamdane, C. Lechauve, M.C. Marden, B. Golinelli-Pimpaneau, Acta Crystallogr. Sect. D Biol. Crystallogr. 70, 1005 (2014)

21. D. M. Scholler, M.-Y. R. Wang, B. M. Hoffman, in Methods Enzymol. vol. 52, ed. by S. Fleischer, L. Packer (1978), pp. 487-493

22. M. Ezhevskaya, E. Bordignon, Y. Polyhach, L. Moens, S. Dewilde, G. Jeschke, S. Van Doorslaer, Mol. Phys. 111, 2855 (2013)

23. C.L. Motion, J.E. Lovett, S. Bell, S.L. Cassidy, P.A.S. Cruickshank, D.R. Bolton, R.I. Hunter, H. El Mkami, S. Van Doorslaer, G.M. Smith, J. Phys. Chem. Lett. 7, 1411 (2016)

24. M.G. Dal Farra, S. Richert, C. Martin, C. Larminie, M. Gobbo, E. Bergantino, C.R. Timmel, A.M. Bowen, M. Di Valentin, ChemPhysChem 20, 931 (2019)

25. I. Beletskaya, V.S. Tyurin, A.Y. Tsivadze, R. Guilard, C. Stern, Chem. Rev. 109, 1659 (2009)

26. T. Tanaka, A. Osuka, Chem. Soc. Rev. 44, 943 (2015)

27. A. Toffoletti, Z. Wang, J. Zhao, M. Tommasini, A. Barbon, Phys. Chem. Chem. Phys. 20, 20497 (2018)

28. A. Regev, S. Michaeli, H. Levanon, M. Cyr, J.L. Sessler, J. Phys. Chem. 95, 9121 (1991)

29. I.V. Borovykh, I.B. Klenina, I.I. Proskuryakov, P. Gast, A.J. Hoff, in Spectroscopy Biology Molecular New Directions. ed. by J. Greve, G.J. Puppels, C. Otto (Springer, Netherlands, 1999), pp. 189-190

30. M. Gouterman, G.H. Wagnière, L.C. Snyder, J. Mol. Spectrosc. 11, 108 (1963)

31. M. Gouterman, J. Chem. Phys. 52, 3795 (1970) 
32. J. H. van der Waals, W. G. van Dorp, T. J. Schmaafsma, in The Porphyrins, ed. by D. Dolphin (Elsevier, Amsterdam, 1979), pp. 257-312

33. M. G. Dal Farra, C. Martin, El. Bergantino, Y. E. Kandrashkin, A. van der Est, M. Di Valentin, Phys. Chem. Chem. Phys. 22, 19982 (2020)

34. Y. E. Kandrashkin, M. Di Valentin, A. van der Est, J. Chem. Phys. 153, 094304 (2020)

35. A. Barbon, M. G. Dal Farra, S. Ciuti, M. Albertini, L. Bolzonello, L. Orian, M. Di Valentin, J. Chem. Phys. 152, 034201 (2020)

36. S. Richert, C.E. Tait, C.R. Timmel, J. Magn. Reson. 280, 103 (2017)

37. C.W.M. Kay, M. Di Valentin, K. Möbius, Sol. Energy Mater. Sol. Cells 38, 111 (1995)

38. L. Bolzonello, M. Albertini, E. Collini, M. Di Valentin, Phys. Chem. Chem. Phys. 19, 27173 (2017)

39. B.M. Hoffman, J. Am. Chem. Soc. 97, 1688 (1975)

40. M. Nappa, J.S. Valentine, J. Am. Chem. Soc. 100, 5075 (1978)

41. Y. Shibata, T. Kushida, Chem. Phys. Lett. 284, 115 (1998)

42. J.A. Wyer, C.S. Jensen, S. Brøndsted Nielsen, Int. J. Mass Spectrom. 308, 126 (2011)

43. J.S. Ahn, Y. Kanematsu, Y. Nishikawa, T. Kushida, Synth. Met. 71, 1735 (1995)

44. T. Hashimoto, Y.-K. Choe, H. Nakano, K. Hirao, J. Phys. Chem. A 103, 1894 (1999)

45. A.J. Redman, G. Moise, S. Richert, E.J. Viere, W.K. Myers, M.J. Therien, C.R. Timmel, J. Phys. Chem. C 125, 11782 (2021)

46. C.E. Tait, P. Neuhaus, H.L. Anderson, C.R. Timmel, J. Am. Chem. Soc. 137, 6670 (2015)

47. M.C. Thurnauer, J.R. Norris, Chem. Phys. Lett. 47, 100 (1977)

48. F. Lendzian, R. Bittl, A. Telfer, W. Lubitz, Biochim. Biophys. Acta Bioenerg. 1605, 35 (2003)

49. A. Agostini, D.M. Palm, F.-J. Schmitt, M. Albertini, M. Di Valentin, H. Paulsen, D. Carbonera, Sci. Rep. 7, 7504 (2017)

50. M. Di Valentin, E. Meneghin, L. Orian, A. Polimeno, C. Büchel, E. Salvadori, C.W.M. Kay, D. Carbonera, Biochim. Biophys. Acta Bioenerg. 1827, 1226 (2013)

51. M. Di Valentin, M. Albertini, M.G. Dal Farra, E. Zurlo, L. Orian, A. Polimeno, M. Gobbo, D. Carbonera, Chem. A Eur. J. 22, 17204 (2016)

Publisher's Note Springer Nature remains neutral with regard to jurisdictional claims in published maps and institutional affiliations. 